E. Alpert • A. Gruzman - Y. Riahi - R. Blejter •

P.Aharoni - G. Weisinger - J. Eckel $\cdot$ N. Kaiser •

S. Sasson

\title{
Delayed autoregulation of glucose transport in vascular endothelial cells
}

Received: 26 January 2004 / Accepted: 7 November 2004 / Published online: 1 March 2005

(C) Springer-Verlag 2005

\begin{abstract}
Aims/hypothesis: We aimed to characterise the development of autoregulation of glucose transport in vascular endothelial cells and its relationship to 12-lipoxygenase (12-LO) expression. Methods: Bovine aortic endothelial cells were exposed to 5.5 and $23.0 \mathrm{mmol} / 1$ glucose for up to $48 \mathrm{~h}$. The rates of glucose transport, GLUT-1 and 12-LO expression and of 12-hydroxyeicosatetraenoic acid (12-HETE) production were determined. Results: We showed high glucose-dependent downregulation of glucose transport and transporter in vascular endothelial cells within $36-48 \mathrm{~h}$. A similar time-dependent increase in the expression of 12-LO and the generation of its product 12-HETE was also observed. This downregulatory process was prevented when lipoxygenase activity was inhibited. Conclusions/interpretation: Vascular endothelial cells, which were previously thought to be "glucose-blind", do in fact downregulate GLUT-1 expression and the rate of glucose transport in response to extended exposure to high glucose concentrations. This slow development of glucose-induced downregulation in vascular endothelial cells is related to the slower basal rate of glucose transport in these cells and the slow induction of 12-LO.
\end{abstract}

E. Alpert · A. Gruzman · Y. Riahi · R. Blejter · P. Aharoni

S. Sasson $(\bowtie)$

Department of Pharmacology,

Hebrew University School of Medicine,

P.O. Box 12272h, Jerusalem, 91120, Israel

e-mail: sassolo@cc.huji.ac.il

Tel: + +972-2-6758798

Fax: +972-2-6758741

G. Weisinger

Institute of Endocrinology, Metabolism and Hypertension,

Tel Aviv Sourasky Medical Center,

Tel Aviv, Israel

J. Eckel

The German Diabetes Research Institute,

Düsseldorf, Germany

N. Kaiser

Endocrinology \& Metabolism Service, Internal Medicine Department, Hadassah-Hebrew University Medical Center, Jerusalem, Israel
These data are interesting in view of current hypotheses that attribute vascular endothelial cell dysfunction in diabetes to the lack of a glucose-induced autoregulatory response.

Keywords Endothelial cells · Glucose transport - Glucose transporter · Hydroxyeicosatetraenoic acids .

Lipoxygenase $\cdot$ Lipoxygenase inhibitors $\cdot$ Vascular smooth muscle

Abbreviations dGlc: 2-deoxy-D-glucose $\cdot\left[{ }^{3} \mathrm{H}\right] \mathrm{dGlc}$ : 2-[1,2- $\left.{ }^{3} \mathrm{H}(\mathrm{N})\right]$ deoxy-D-glucose · GLUT-1: Glucose transporter 1 - HETE: Hydroxyeicosatetraenoic acid . HPLC: High-performance liquid chromatography - LO: Lipoxygenase - 12-LO: 12-lipoxygenase - VEC: Vascular endothelial cells · VSMC: Vascular smooth muscle cells

\section{Introduction}

We reported previously that vascular smooth muscle cells (VSMC), but not vascular endothelial cells (VEC), could downregulate their rate of glucose transport when exposed to a short-term high-glucose challenge $[1,2]$. Other investigators suggest that VEC are particularly susceptible to the deleterious effects of high glucose levels due to an unregulated glucose transport system [3, 4]. We recently reported that VEC that had been exposed to high glucose levels for $48 \mathrm{~h}$ downregulated the rate of glucose transport by reducing glucose transporter-1 (GLUT-1) mRNA and protein expression, as well as GLUT-1 plasma membrane localisation [5]. The present study was undertaken to characterise the development of the autoregulatory response in VEC and its relationship to 12-lipoxygenase (12-LO) induction and function. The results are discussed in view of current hypotheses on the causes of VEC dysfunction in diabetes.

\section{Materials and methods}

Reagents DMEM and sera were from Biological Industries (Kibbutz Beth-Haemek, Israel). American Radiolabelled 
Chemicals (St. Louis, MO, USA) supplied 2-[1,2- $\left.{ }^{3} \mathrm{H}(\mathrm{N})\right]$ deoxy-D-glucose $\left.\left(2,22 \mathrm{TBq} / \mathrm{mmol} ;{ }^{3} \mathrm{H}\right] \mathrm{dGlc}\right)$. Bovine fibronectin, esculetin, 2-deoxy-D-glucose (dGlc) and D-glucose were from Sigma Chemicals (St. Louis, MO, USA). Baicalein (5,6,7-trihydroxyflavonate) was from Aldrich (Milwaukee, MI, USA). Cayman Chemical (Ann Arbor, MI, USA) supplied 5-, 12- and 15-hydroxyeicosatetraenoic acid (HETE) and purified porcine leucocyte-type 12-LO.

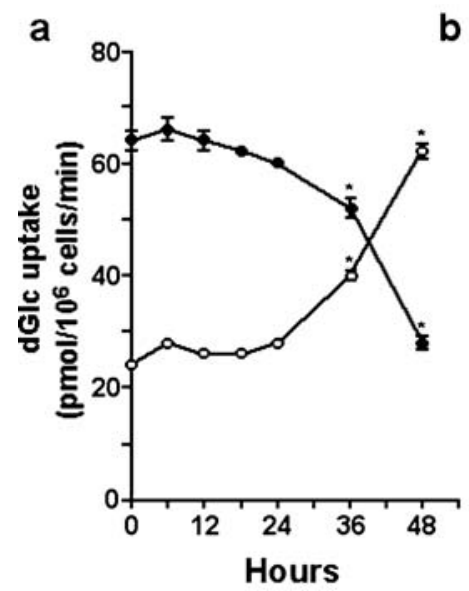

C

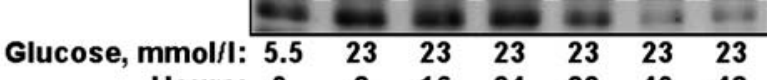

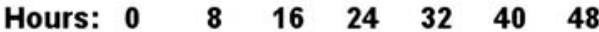

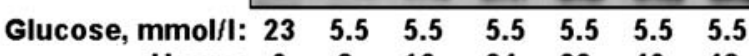

Hours: $\begin{array}{lllllll}0 & 8 & 16 & 24 & 32 & 40 & 48\end{array}$

VSMC

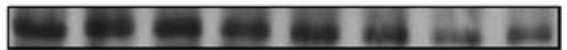

Glucose, mmol/l: $\begin{array}{llllllll}5.5 & 23 & 23 & 23 & 23 & 23 & 23 & 23\end{array}$

Hours: $\begin{array}{llllllll}0 & 3 & 6 & 9 & 12 & 16 & 24 & 36\end{array}$

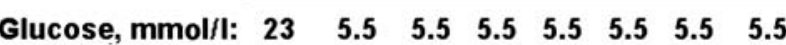

Hours: $0 \begin{array}{llllllll}0 & 3 & 6 & 9 & 12 & 16 & 24 & 36\end{array}$

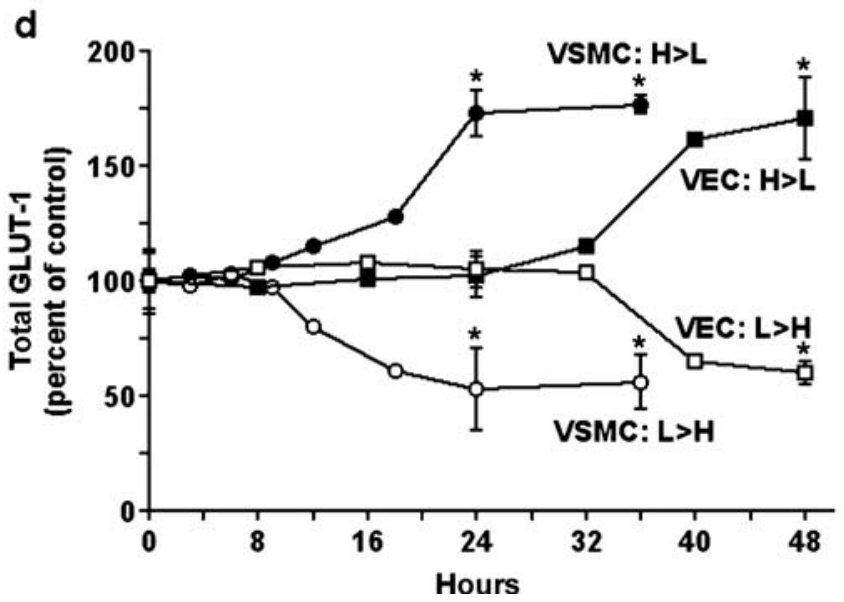

Vascular cell cultures Primary cultures of bovine aortic endothelial and smooth muscle cells were prepared and characterised as described previously [5].

Hexose uptake assay The $\left[{ }^{3} \mathrm{H}\right] \mathrm{dGlc}$ uptake assay was performed as described [1].

Western blot analysis of GLUT-1and 12-lipoxygenase Cell lysates were prepared as described [1]. Western blot analysis of GLUT-1 was performed as described [5]. Western blot analysis of $12-\mathrm{LO}$ was performed using rabbit polyclonal antibody against a synthetic 24-amino acid polypeptide designed from the exon 4 domain of human platelet-type 12-LO, as described [6]. The corresponding DNA sequence for this peptide shares $71 \%$ homology with bovine leucocyte-type 12-LO, and the generated antibodies recognise this protein.

Extraction of hydroxyeicosatetraenoic acids from culture medium and high-performance liquid chromatography analysis Cell culture media were collected from three culture plates for each data point $(2 \mathrm{ml} / \mathrm{well})$, pooled, extracted and analysed for 12-HETE by high-performance liquid chromatography (HPLC), as described previously [5]. The yield and retention time of 12-HETE were $93 \%$ and $11.4 \mathrm{~min}$, respectively.

Statistical analysis Statistical analysis was done using the Mann-Whitney test.

\section{Results}

Time-dependent autoregulation of glucose transport and GLUT-1 expression in vascular cells

Both VEC and VSMC reduced their rates of hexose transport by about $60 \%$ following a 48 -h conditioning period at $23.0 \mathrm{mmol} / 1$ glucose, in comparison with $5.5 \mathrm{mmol} / \mathrm{l}$ glucose incubation at $0 \mathrm{~h}$ (Fig. 1a, b). The cultures were washed and received fresh medium supplemented with the opposite glucose concentration and the rates of hexose uptake were determined at the indicated times. As shown before, VSMC down- or upregulated maximally the rate of glucose transport within $24 \mathrm{~h}$ exposure to 23.0 and $5.5 \mathrm{mmol} / 1$ glucose, respectively $[1,5]$. VEC started to regulate the rate of hexose

Fig. 1 Time- and glucose-dependent autoregulation of hexose transport and GLUT-1 expression in VEC and VSMC. a Confluent VEC, and $\mathbf{b}$ VSMC cultures that had been pre-exposed to 5.5 or $23.0 \mathrm{mmol} / \mathrm{l}$ glucose for $48 \mathrm{~h}$ received fresh medium containing the opposite glucose concentrations. The rate of $\left[{ }^{3} \mathrm{H}\right] \mathrm{dGlc}$ uptake was determined at the indicated times. Closed symbols: 5.5 to $23.0 \mathrm{mmol} / \mathrm{l}$ glucose transfer at time zero; open symbols: 23.0 to $5.5 \mathrm{mmol} / 1$ glucose transfer. cWestern blot analysis of GLUT-1 $\left(45 \mathrm{M}_{\mathrm{r}}\right)$ content in whole-cell lysates of VEC and VSMC, which were treated similarly. $\mathbf{d}$ Relative changes in total GLUT-1, determined by densitometric analysis. The $100 \%$ values were taken at $0 \mathrm{~h}$ for each respective treatment. Values are means \pm SEM, $n=3$, where SEM bars are depicted. ${ }^{*} p<0.05$ for difference from the respective control (at $0 \mathrm{~h}$ ) 
transport only after a 24 -h delay, with the maximal down- or upregulatory response observed by $48 \mathrm{~h}$. Control cells, maintained at the original glucose concentration throughout this incubation period, did not significantly modify their respective rates of hexose transport (data not shown). Figure 1c and d shows a similar time- and glucose-dependent up- or downregulation of GLUT-1 expression in cells that were transferred from 23.0 to $5.5 \mathrm{mmol} / 1$ glucose, or vice versa, respectively.
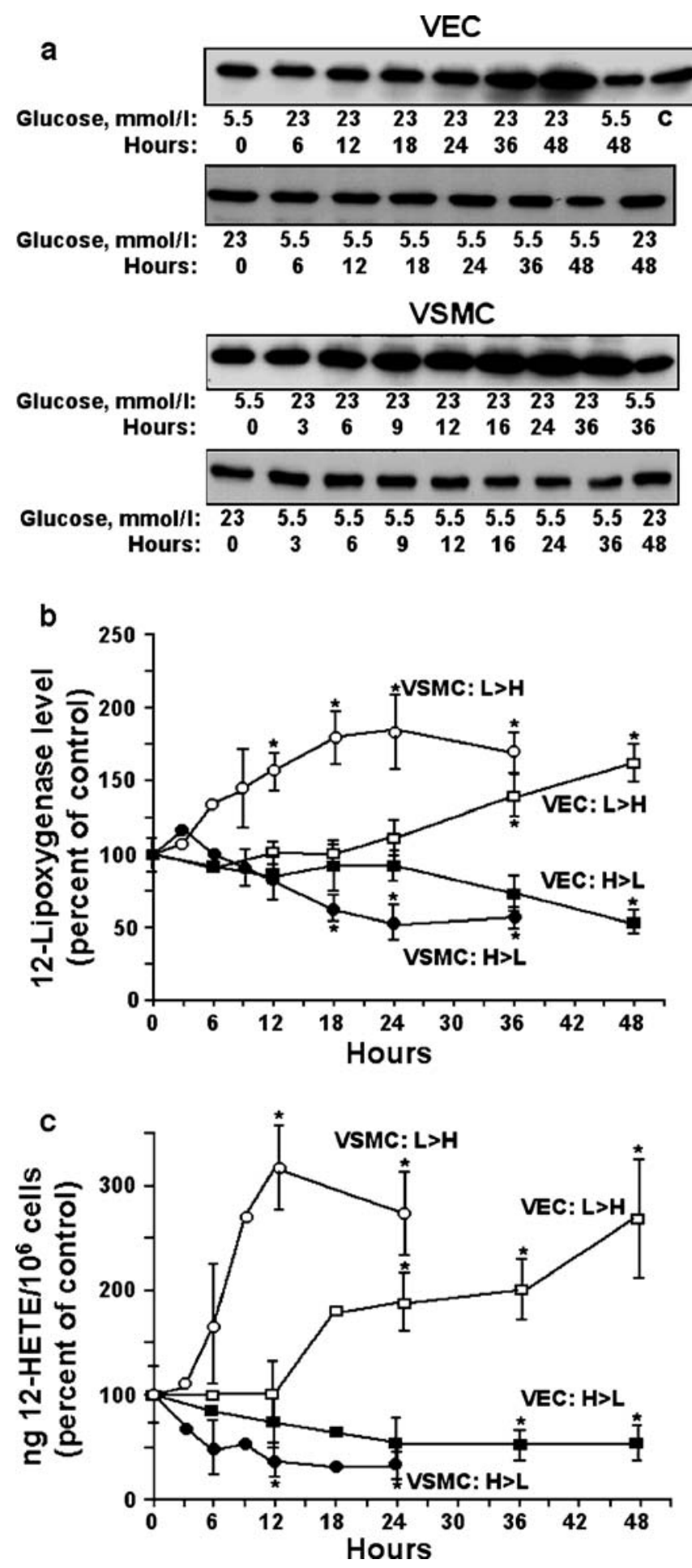

We had previously shown that lipoxygenase inhibitors, and particularly 12-LO inhibition, preserved or prevented high glucose-induced downregulation of glucose transport in vascular cells [5]. When tested in the present study, esculetin (a general lipoxygenase inhibitor) and baicalein (a 12-LO inhibitor) prevented high-glucose-dependent downregulation of glucose transport in VEC and VSMC. We determined the rates of hexose uptake in VEC and VSMC that had been exposed for 48 or $24 \mathrm{~h}$, respectively, to $5.5,23.0$ or $23.0 \mathrm{mmol} / \mathrm{l}$ glucose plus $100 \mu \mathrm{mol} / \mathrm{l}$ esculetin or $100 \mu \mathrm{mol} / \mathrm{l}$ baicalein. In VEC the rates were: $71 \pm 11,39 \pm 8,83 \pm 9$ and 78 $\pm 2 \mathrm{pmol} \mathrm{dGlc} / 10^{6}$ cells/min, respectively. In VSMC the respective values were: $184 \pm 12,103 \pm 6,215 \pm 17$ and 185 $\pm 9 \mathrm{pmol} \mathrm{dGlc} / 10^{6}$ cells $/$ min (mean \pm SEM, $n=3$ ).

The rate of hexose transport in cells exposed to $5.5 \mathrm{mmol} / \mathrm{l}$ glucose plus $17.5 \mathrm{mmol} / \mathrm{l}$ sucrose was similar to the rate in cells exposed to $5.5 \mathrm{mmol} / \mathrm{l}$ glucose (VEC: $74 \pm 6$ and $69 \pm 5$, respectively; VSMC: $193 \pm 12$ and $189 \pm 9 \mathrm{pmol} \mathrm{dGlc} / 10^{6}$ cells/min, respectively; means $\pm \mathrm{SEM}, n=3$ ), indicating that the slightly increased osmolarity of the high glucose medium did not alter the transport system. It should be noted that the rate of glucose transport in VEC was about $30 \%$ of that measured in VSMC at $0 \mathrm{~h}$ and end points (Fig. 1a, b).

Glucose- and time-dependent regulation of 12-LO expression and activity

VEC and VSMC increased or decreased 12-LO expression in a time-dependent manner when transferred from 5.5 to $23.0 \mathrm{mmol} / \mathrm{l}$ glucose or vice versa (Fig. 2a, b). The changes in 12-LO expression in VEC were observed after a 24-h delay, being maximal by $48 \mathrm{~h}$, whereas maximal changes in VSMC were already apparent by 18 to $24 \mathrm{~h}$.

Similar glucose- and time-dependent changes in 12-HETE secretion from VEC and VSMC were also observed (Fig. 2c). A close correlation exists among these temporal changes of the amounts of secreted 12-HETE, 12-LO expression (Fig. 2a, b), and the changes in GLUT-1 expression and the rate of glucose transport in VEC and VSMC (Fig. 1a-d).

\section{Discussion}

Vascular endothelial cells have been described as 'glucoseblind', due to their inability to downregulate the rate of glu-

Fig. 2 Glucose- and time-dependent expression and activity of 12LO in VEC and VSMC. Confluent VSMC or VEC cultures were pre-exposed to $5.5 \mathrm{mmol} / 1$ and then to $23.0 \mathrm{mmol} / 1$ glucose or vice versa (see legend, Fig. 1). a A representative Western blot analysis of 12-LO. C, purified porcine leucocyte-type 12-LO (72 kDa), which served as a positive control. b Relative changes (to $0 \mathrm{~h}$ ) in 12LO expression, determined by densitometric analysis. c Culture media were pooled from three culture plates of similar VSMC or VEC and extracted. The amounts of 12-HETE in the extracts were determined by HPLC analysis. The $100 \%$ values at $0 \mathrm{~h}$ were (in $\mathrm{ng} / 10^{6}$ cells): 5.3 and 16.1 for VEC maintained at 5.5 and $23.0 \mathrm{mmol} /$ 1 glucose, respectively, and 26.2 and 57.6 for VSMC, respectively. L, $5.5 \mathrm{mmol} / 1$ glucose, $\mathrm{H}, 23.0 \mathrm{mmol} / 1$ glucose. Values are means \pm SEM, $n=3$, where SEM bars are depicted. ${ }^{*} p<0.05$ 
cose transport when exposed to high glucose levels for $24 \mathrm{~h}$ $[1,2]$. These early observations have led other investigators to suggest that VEC are unique in their inability to modify glucose transport when exposed to extracellular high glucose concentrations, and thant an unregulated influx of high glucose levels causes overproduction of reactive oxygen species, which consequently impair VEC functions $[3,4,7,8]$. The present data show that, in response to changes in ambient glucose, VEC do in fact reversibly regulate GLUT-1 expression and their hexose transport system in a lipoxygenase-dependent manner. The respective changes in GLUT-1 expression, glucose transport capacity, 12-LO expression and 12-HETE production share a similar time dependency.

The delayed response in the initiation of high glucosedependent downregulation of glucose transport in VEC is attributed to: (1) the low rate of glucose uptake that may lead to a slow accumulation of glucose metabolites, which transduce the downregulatory response; and (2) a parallel slow induction of 12-LO and generation of 12-HETE. Yet, once the process has started, it exhibits a comparable rate of progression in VEC and VSMC, reaching the end point $24 \mathrm{~h}$ after initiation. We have reported before that inhibition of 12-LO prevented glucose-induced regulation of GLUT-1 mRNA and protein expression and GLUT-1 localisation to the plasma membrane in vascular cells [5]. The present data further support the hypothesis that 12-HETE mediates glucosedependent downregulation in VEC and VSMC.

This study indicates that the downregulatory response may protect VEC against deleterious effects of increased glucose influx during long-term or chronic hyperglycaemic conditions. However, short intermittent periods of hyperglycaemia could leave VEC vulnerable to such deleterious effects of an uncontrolled increase in intracellular glucose levels. Thus, particular attention should be given to the duration of exposure of VEC to high glucose levels.

Various types of endothelial cells may respond differently to high glucose concentrations. For instance, bovine retinal endothelial cells maintained a constant level of glucose uptake throughout a 72-h incubation period at either 5.0 or $30.0 \mathrm{mmol} / 1$ glucose, while bovine brain and rat heart endothelial cells already downregulated glucose transport within $24 \mathrm{~h}$ of exposure to $30.0 \mathrm{mmol} / \mathrm{l}$ glucose [9]. Thus, various types of VEC, such as macro- and microvascular, may respond differently to hyperglycaemic conditions.
In conclusion, acute and prolonged high glucose challenges affect bovine aortic endothelial cells differently. While, under the former condition, the cells are exposed to the full array of harmful effects of unrestricted high glucose influx, in the latter case, the cells are partially protected due to downregulation of hexose transport.

Acknowledgements S. Sasson is a member of The David R. Bloom Center for Pharmacy at the Hebrew University. E. Alpert and A. Gruzman received fellowships from The Hebrew University Center for Diabetes Research. This work was supported by grants from The Yedidut Foundation Mexico, The German-Israel Foundation for Scientific Research \& Development (I-750-165.2/2002), and the Israel Ministry of Health.

\section{References}

1. Kaiser N, Sasson S, Feener E et al (1993) Differential regulation of glucose transport and transporters by glucose in vascular endothelial and smooth muscle cells. Diabetes 42:80-89

2. Sasson S, Gorowitz N, Joost HG, King GL, Cerasi E, Kaiser N (1996) Regulation by metformin of the hexose transport system in vascular endothelial and smooth muscle cells. Br J Pharmacol 177:1318-1324

3. Hammes H-P, Du X, Edelstein D et al (2003) Benfotiamine blocks three major pathways of hyperglycemic damage and prevents experimental diabetic retinopathy. Nat Med 9:294-299

4. Creager MA, Lüscher TF (2003) Diabetes and vascular disease: Pathophysiology, clinical consequences, and medical therapy: part 1. Circulation 108:1527-1532

5. Alpert E, Gruzman A, Totary H, Kaiser N, Reich R, Sasson S (2002) A natural protective mechanism against hyperglycemia in vascular endothelial and smooth-muscle cells: role of glucose and 12-hydroxyeicosatetraenoic acid. Biochem J 362:413-422

6. Limor R, Weisinger G, Gilad S et al (2001) A novel form of platelet-type 12-lipoxygenase mRNA in human vascular smooth muscle cells. Hypertension 38:864-871

7. Guzik TJ, Mussa S, Gastaldi D et al (2002) Mechanisms of increased vascular superoxide production in human diabetes mellitus: role of $\mathrm{NAD}(\mathrm{P}) \mathrm{H}$ oxidase and endothelial nitric oxide synthase. Circulation 105:1656-1662

8. Milstien S, Katusic Z (1999) Oxidation of tetrahydrobiopterin by peroxynitrite: implications for vascular endothelial function. Biochem Biophys Res Commun 263:681-684

9. Rajah TT, Olson AE, Grammas P (2001) Differential glucose uptake in retina and brain-derived endothelial cells. Microvasc Res 62:236-242 